Gynäkologe 2018 ·1:286-295

https://doi.org/10.1007/s00129-018-4205-9

Online publiziert: 2. Februar 2018

(c) Der/die Autor(en) 2018. Dieser Artikel ist

eine Open-Access-Publikation.

\section{Redaktion}

T. Strowitzki, Heidelberg

R. Kuon, Heidelberg

CrossMark

\author{
S. Rudnik-Schöneborn ${ }^{1} \cdot$ M. Swoboda $\cdot$ J. Zschocke \\ 'Sektion Humangenetik, Medizinische Universität Innsbruck, Innsbruck, Österreich \\ ${ }^{2}$ VivaNeo Kinderwunschklinik Dr. Loimer, Wels, Österreich
}

\title{
Genetische Untersuchungen bei wiederholten Spontanaborten
}

\section{Aktuelle Empfehlungen unter besonderer Berücksichtigung der Präimplantationsdiagnostik}

Fehlgeburten betreffen $15 \%$ aller Schwangerschaften und nehmen mit steigendem mütterlichem Alter deutlich an Häufigkeit zu. Der Wunsch nach einer Ursachenklärung ist groß, ebenso wie der Wunsch nach einer ärztlichen Intervention, um die Erfolgsraten für weitere Schwangerschaften zu verbessern. Der Leidensdruck der Paare bewirkt, dass Ärztinnen und Ärzte unterschiedliche Behandlungsstrategien anbieten, die teilweise wenig bis nicht evidenzbasiert sind. Dieser Artikel hat das Ziel, den aktuellen Kenntnisstand zur Frage der genetischen Diagnostik bei Paaren mit Aborten darzustellen, unter besonderer Berücksichtigung des Aneuploidiescreenings zur Verbesserung der Schwangerschaftsund Lebendgeburtenraten. Es werden die rechtlichen Rahmenbedingungen im deutschen Sprachraum skizziert und die Empfehlungen internationaler Leitlinien zur genetischen Diagnostik und Therapie von wiederholten Spontanaborten miteinander verglichen. Hier berücksichtigt werden Leitlinien der deutschsprachigen Fachgesellschaften (Deutsche Gesellschaft für Gynäkologie und Geburtshilfe, DGGG; Arbeitsgemeinschaft für Immunologie in der Gynäkologie und Geburtshilfe, AGIM; [20]), der European Society of Human Reproduction and Embryology, ESHRE [11], des Royal College of Obstetricians and Gynaecologists, RCOG [31], des American College of Obstetricians and Gynecologists, ACOG [1] und der Amer- ican Society for Reproductive Medicine, ASRM [2].

》) Schon ab einem Abort steigt
die Wahrscheinlichkeit einer
familiären Chromosomenverän-
derung

Die WHO(World Health Organization)Definition des wiederholten Spontanabortes lautet: „3 und mehr konsekutive Fehlgeburten vor der 20. SSW“ [38]. Hiervon sind etwa $1 \%$ der Paare mit Kinderwunsch betroffen [5]. Dieser Definition haben sich die britische (RCOG; [31]) und die deutschen Fachgesellschaften [20] angeschlossen; sie empfehlen eine weiterführende Ursachenabklärung ab 3 Aborten. Dies ist aus genetischer Sicht zu relativieren, da bereits ab einem Abort die Wahrscheinlichkeit einer familiären Chromosomenveränderung ansteigt. Berücksichtigt man Paare mit mindestens 2 Aborten für die genetische Diagnostik, sind bis zu $5 \%$ aller Paare im reproduktionsfähigen Alter betroffen [5].

\section{Genetische Ursachen von Aborten}

\section{Chromosomenstörungen im Abortmaterial}

Die häufigste Ursache (40-50\%) für Spontanaborte sind embryonale bzw. fetale Chromosomenaberrationen, die mehrheitlich in der elterlichen Keimbahn bzw. in der Fruchtanlage neu entstanden sind. Je früher ein Abort eintritt, desto wahrscheinlicher ist das Vorliegen einer embryonalen/fetalen Chromosomenstörung. So lassen sich im ersten Trimenon in etwa $50 \%$ der Aborte Chromosomenaberrationen nachweisen, während die Rate im zweiten Trimenon nur noch bei etwa $30 \%$ liegt [16]. Nach Daten einer systematischen Übersicht [35] beträgt die Prävalenz einer Chromosomenaberration bei einem Spontanabort $45 \%$ und sinkt geringfügig auf $39 \%$ bei Frauen mit mindestens 2 vorangegangenen Aborten. Diese Daten entsprechen auch einer japanischen Studie [29], die eine signifikante Abnahme von abnormen Karyotypen in Abortmaterial mit zunehmender Anzahl von Aborten ermittelte (• Tab. 1). Der Anteil von Chromosomenstörungen sank von $65,6 \%$ nach 2 Aborten auf $28,6 \%$ bei Frauen mit 6 Aborten in einer Altersgruppe von durchschnittlich 29-32 Jahren [29]. Ein normaler Karyotyp im Abortmaterial wurde als Prädiktor für einen weiteren Abort in folgenden Schwangerschaften gewertet. Der Anteil der nummerischen Chromosomenaberrationen an der Gesamtzahl der Chromosomenaberrationen ist bei einem Abort oder nach mehreren Aborten mit ca. $90 \%$ praktisch identisch [35].

Bei Frühaborten erfolgt in der Praxis - unabhängig, ob sie einer medikamentösen Abortinduktion oder einer Abort- 
Tab. 1 Normaler Karyotyp im Abortmaterial in Abhängigkeit von der Anzahl vorangegangener Aborte bei Paaren mit mindestens 2 vorausgegangenen Aborten und normalem elterlichen Karyotyp. (Mod. nach Ogasawara et al. [29])

\begin{tabular}{|c|c|c|c|}
\hline $\begin{array}{l}\text { Vorausgegangene } \\
\text { Aborte }\end{array}$ & $\begin{array}{l}\text { Normaler } \\
\text { Karyotyp* (\%) }\end{array}$ & Abortrate (\%) & $\begin{array}{l}\text { Mütterliches Alter } \\
\text { (Jahre) }\end{array}$ \\
\hline 2 & 36,4 & 23,2 & $29,4 \pm 3,8$ \\
\hline 3 & 41,0 & 32,4 & $30,6 \pm 3,6$ \\
\hline 4 & 44,7 & 40,0 & $31,4 \pm 3,9$ \\
\hline 5 & 61,1 & 48,7 & $32,5 \pm 3,6$ \\
\hline 6 & 71,4 & 64,1 & $32,8 \pm 4,1$ \\
\hline
\end{tabular}

Tab. 2 Risiko für wiederholte Fehlgeburten in Abhängigkeit vom maternalen Alter und der Anzahl vorangegangener Aborte. (Nach Nybo-Andersen et al. [28])

\begin{tabular}{lllll}
$\begin{array}{l}\text { Vorangegangene } \\
\text { Aborte }\end{array}$ & \multicolumn{2}{l}{ Wiederholungsrisiko (\%) } & \\
& 25-29 Jahre & 30-34 Jahre & 35-39 Jahre & 40-44 Jahre \\
\hline 1 Abort & 15 & $16-18$ & $21-23$ & 40 \\
\hline 2 Aborte & $22-24$ & $23-26$ & $25-30$ & $40-44$ \\
$\geq 3$ Aborte & $40-42$ & $38-40$ & $40-45$ & $60-65$
\end{tabular}

Tab. 3 Anstieg von Trisomien in Spontanaborten mit zunehmendem mütterlichen Alter. (Nach Eiben et al. [9])

\begin{tabular}{|l|l|l|l|l}
$\begin{array}{l}\text { Alter der Mut- } \\
\text { ter (Jahre) }\end{array}$ & $\begin{array}{l}\text { Chromosomenstörungen } \\
\text { insgesamt (\%) }\end{array}$ & $\begin{array}{l}\text { Trisomien } \\
\text { (\%) }\end{array}$ & $\begin{array}{l}\text { Polyploidie } \\
\text { (\%) }\end{array}$ & $\begin{array}{l}\text { Monosomie X } \\
\text { (\%) }\end{array}$ \\
\hline$<25$ & 40,0 & 14,3 & 12,9 & 10,0 \\
\hline $25-29$ & 40,5 & 21,5 & 12,8 & 4,6 \\
\hline $30-34$ & 55,4 & 37,5 & 11,4 & 3,8 \\
\hline$\geq 35-39$ & 67,8 & 48,3 & 10,2 & 5,9 \\
\hline$>39$ & 82,2 & 80 & 0 & 0
\end{tabular}

kürettage zugeführt werden - meist keine genetische Diagnostik des Abortgewebes. Indikationen für eine genetische Analyse des Abortmaterials können sonographische Hinweise auf fetale Entwicklungsstörungen sein. Besteht aufgrund des kindlichen Fehlbildungsmusters bei einem Abort der Verdacht auf eine Chromosomenstörung, sollte zunächst eine klassische (lichtmikroskopische) Chromosomenanalyse erfolgen. Dabei ist sicherzustellen, dass die Qualitätskriterien hinsichtlich der Bandenauflösung und der Zahl der untersuchten Metaphasen erfüllt sind und dass die Untersuchung je nach Fragestellung durch zusätzliche Methoden (z.B. Fluoreszenz-in-situHybridisierung, FISH) ergänzt werden kann [8]. Bei unauffälligem Befund der klassischen Chromosomenanalyse sollte eine DNA-Array-Analyse (molekulare Karyotypisierung) im Abortgewebe in Betracht gezogen werden, da hierdurch auch kleinere Aberrationen (Deletionen und Duplikationen) erfasst werden, die bei der konventionellen Chromosomenanalyse nicht detektiert werden können. Für die klassische Chromosomenanalyse wird embryonales oder fetales Gewebe benötigt, das kultiviert werden muss, während eine Array-Analyse aus einer DNA-Probe möglich ist.

Bei der zytogenetischen Diagnostik von Abortmaterial ist zu bedenken, dass die Bandenauflösung meist gering ist (200-300 Banden) und dass bei einem beträchtlichen Anteil keine Chromosomenanalyse mehr gelingt. Der Anteil der Aborte, die nicht mehr entsprechend analysiert werden können, liegt bei ca. $18 \%$ für die klassische Chromosomenanalyse und bei ca. $5 \%$ für eine Array-Analyse [35]. Insgesamt können durch molekularzytogenetische Analysen nur in etwa $5 \%$ der Fälle zusätzliche Chromosomenaberrationen detektiert werden, sodass ein routinemäßiger Einsatz der Array-Analyse zur Klärung von Abortursachen derzeit nicht für sinnvoll gehalten wird [35].

\section{》) Je früher es zu einem Abort kommt, desto wahrscheinlicher ist eine Chromosomenstörung}

Neue Sequenzierungstechnologien werden in der Zukunft wahrscheinlich zur kombinierten Diagnostik chromosomaler und monogener Ursachen von Entwicklungsstörungen zum Einsatz kommen. Bei Nachweis einer strukturellen Chromosomenaberration im Abortmaterial ist eine Chromosomenanalyse bei beiden Elternteilen indiziert, falls diese nicht bereits im Vorfeld erfolgte. Das Ergebnis soll im Rahmen einer genetischen Beratung entsprechend der nationalen gesetzlichen Regelungen mitgeteilt werden.

\section{Alterseffekt}

Das Wiederholungsrisiko für Fehlgeburten schwankt in Abhängigkeit von verschiedenen Faktoren erheblich. Dabei nimmt neben dem Alter der Mutter auch die Anzahl der vorangegangenen Aborte Einfluss (•Tab. 2), während das väterliche Alter für das Abortrisiko statistisch keine Rolle spielt. Der Anteil der Mütter, die bei der Geburt ihres Kindes über 35 Jahre alt waren, hat sich in Deutschland seit 1991 etwa vervierfacht und lag 2012 bei $22 \%$ (Statistisches Bundesamt 2012). Mit zunehmendem mütterlichem Alter steigt insbesondere das Risiko für embryonale/fetale autosomale Trisomien (- Tab. 3). Diese lassen sich bei 60-70\% der zytogenetisch auffälligen Spontanaborte nachweisen [35]. Am häufigsten zeigt sich bei Aborten die Trisomie 16, gefolgt von den Trisomien 22, 15 und 21 [33]. Polyploidien finden sich bei etwa 15-20\%, eine Monosomie X bei 10-15\% der zytogenetisch auffälligen Aborte [33, 35]. Für die Monosomie X und strukturelle Chromosomenaberrationen ist kein Zusammenhang mit dem mütterlichen Alter erkennbar [9, 30].

Entsprechend der Zahl der Chromosomenfehlverteilungen in den Oozyten 
Gynäkologe 2018·51:286-295 https://doi.org/10.1007/s00129-018-4205-9

(c) Der/die Autor(en) 2018. Dieser Artikel ist eine Open-Access-Publikation.

\section{S. Rudnik-Schöneborn $\cdot$ M. Swoboda $\cdot J$. Zschocke}

\section{Genetische Untersuchungen bei wiederholten Spontanaborten. Aktuelle Empfehlungen unter besonderer Berücksichtigung der Präimplantationsdiagnostik}

\section{Zusammenfassung}

Mit $40-50 \%$ stellen embryonale bzw. fetale Chromosomenaberrationen die häufigste Ursache für Spontanaborte dar. Bei etwa $5 \%$ aller Paare treten 2, bei etwa $1 \%$ aller Paare 3 Aborte auf, wobei die Häufigkeit mit dem in Europa anhaltenden Trend zu höherem mütterlichem Alter bei Konzeption weiter zunimmt. Das Wiederholungsrisiko von Aborten steigt mit dem Alter der Mutter und der Anzahl der vorangegangenen Aborte, das väterliche Alter hat statistisch keinen Einfluss. Bei dem sog. Altersrisiko spielen zahlenmäßige Chromosomenveränderungen, insbesondere autosomale Trisomien, die wichtigste Rolle. Bei etwa 4-5\% der Paare mit mindestens 2 Aborten ist ein Elternteil
Träger einer balancierten Chromosomenveränderung (Translokation, Inversion), die mit erhöhten Risiken für die Geburt eines Kindes mit Entwicklungsstörungen einhergeht und ein gezieltes Angebot einer Pränatalbzw. Präimplantationsdiagnostik nach sich zieht. Dargestellt werden die Empfehlungen nationaler und internationaler Leitlinien zur genetischen Diagnostik und Therapie von wiederholten Spontanaborten, verglichen werden die rechtlichen Rahmenbedingungen für die Pränatal- und Präimplantationsdiagnostik im deutschen Sprachraum, d.h. in Deutschland, Österreich und in der Schweiz. Mehrheitlich wird eine Chromosomenanalyse der Eltern nach wiederholten Aborten empfohlen, während weitere genetische Analysen - z. B. ein Thrombophiliescreening - derzeit nicht für sinnvoll gehalten werden. Die Hoffnung, dass ein Aneuploidiescreening vor Implantation bei Paaren mit wiederholten Aborten zu einer besseren Schwangerschaftsund Lebendgeburtenrate führt, hat sich bislang nicht erfüllt. Aktuell wird eine Präimplantationsdiagnostik bei Paaren mit wiederholten Aborten ohne Fertilitätsstörung von keiner Fachgesellschaft empfohlen.

\section{Schlüsselwörter}

Chromosomale Aberrationen · Mütterliches Alter · Karyotypisierung · Pränataldiagnostik . Thrombophilie

\section{Genetic testing in recurrent spontaneous miscarriages. Current recommendations taking into consideration preimplantation genetic testing}

\section{Abstract}

Embryonic or fetal chromosome aberrations are the most frequent causes of spontaneous abortions and are found in $40-50 \%$ of first trimester miscarriages. About $5 \%$ of couples experience two and about $1 \%$ experience three recurrent miscarriages. This incidence is expected to further increase with increasing maternal age in European countries. Recurrence risk for early miscarriages is correlated with maternal age and number of previous miscarriages, while paternal age has no significant impact. The increased risk is mostly due to numerical chromosomal aberrations in oocytes, in particular trisomies. In 4-5\% of couples with recurrent miscarriage a balanced structural chromosome anomaly (transloca- tion, inversion) can be detected in one of the partners. In these cases, future offspring have an increased risk of a developmental defect caused by an unbalanced chromosomal aberration. This has implications with regard to the option of prenatal or preimplantation genetic diagnosis in further pregnancies. In this review we address current guidelines for genetic testing and management of couples with recurrent miscarriages, with particular focus on the legal regulations for prenatal and preimplantation genetic diagnosis in Germanspeaking countries, i. e., Germany, Austria, and Switzerland. Most recommendations include karyotyping of both partners after two or more miscarriages, while analysis of further genetic factors (e. g., screening for thrombophilia) is currently not considered useful. With the implementation of preimplantation genetic screening for chromosomal aberrations in early embryos it was hoped to also improve pregnancy outcome rates for couples with recurrent miscarriages. However, this proved not to be the case so far, and preimplantation genetic testing is still not recommended in these circumstances.

\section{Keywords}

Chromosome aberrations - Maternal age $\cdot$ Karyotyping $\cdot$ Prenatal diagnosis . Thrombophilia korreliert auch die Wahrscheinlichkeit für eine Lebendgeburt wesentlich mehr mit einem jüngeren mütterlichen Alter als mit der Zahl vorangegangener Aborte (- Tab. 4). Die kumulative Wahrscheinlichkeit für eine Lebendgeburt beträgt nach wiederholten Fehlgeburten in der Altersgruppe bis 30 Jahre über $80 \%$, in der Gruppe von 30-34 Jahren ca. 70\%, mit 35-39 Jahren ca. $60 \%$ und sinkt unter $50 \%$ ab einem mütterlichen Alter von 40 Jahren [22].

\section{Strukturelle Chromosomen- störungen bei Aborten}

In den meisten Leitlinien wird eine mikroskopische Chromosomenanalyse (Karyotypisierung) bei beiden Partnern empfohlen, nachdem sich wiederholte Aborte ereignet haben. Vor jeder genetischen Diagnostik muss entsprechend den nationalen Regelungen eine Aufklärung über die geplante Untersuchung durch qualifizierte Ärzte erfolgen. Zusätzlich muss ein schriftliches Einverständnis der Ratsuchenden vorliegen. Die gesetzlichen Grundlagen für genetische Analysen sind in Deutschland durch das Gendiagnostikgesetz (GenDG), in Österreich durch das Gentechnik-Gesetz (GTG) und in der Schweiz durch das Bundesgesetz über genetische Untersuchungen am Menschen (GUMG) geregelt.

Wird bei einem der Partner eine balancierte Chromosomenaberration (Translokation, Inversion) nachgewiesen, ist die chromosomale Anordnung der DNA ver- 
Tab. 4 Wahrscheinlichkeit für eine Lebendgeburt in einem Zeitraum von 5 Jahren nach Erstberatung in Abhängigkeit vom maternalen Alter und von der Anzahl vorangegangener Aborte. (Nach Lund et al. [22])

\begin{tabular}{l|lllll|l}
$\begin{array}{l}\text { Vorausgegangene } \\
\text { Aborte }\end{array}$ & \multicolumn{4}{l}{ Wahrscheinlichkeit für Lebendgeburt nach Altersgruppen (Jahre) } \\
\hline & $\mathbf{2 0 - 2 4}$ & $\mathbf{2 5 - 2 9}$ & $\mathbf{3 0 - 3 4}$ & $\mathbf{3 5 - 3 9}$ & $>\mathbf{4 0}$ \\
\hline 3 & 1,28 & 1,5 & 1 & 0,81 & 0,48 \\
\hline 4 & 1,93 & 0,99 & 0,95 & 0,67 & 0,88 \\
5 & 0,48 & 1,51 & 0,79 & 0,76 & 0,32 \\
$\geq 6$ & k. A. & 0,80 & 0,55 & 0,51 & k.A.
\end{tabular}

k. A. Keine Angabe möglich

Tab. 5 Verteilung von elterlichen Chromosomenanomalien in Abhängigkeit von der Anzahl vorangegangener Aborte, Anzahl ( $n$ ) Patienten in Klammern. (Mod. nach De Braekeleer und Dao [7])

\begin{tabular}{|c|c|c|c|}
\hline Anzahl Aborte & 1 & 2 & $>3$ \\
\hline Gesamtzahl ( $n$ ) der untersuchten Personen & 4224 & 23.416 & 5680 \\
\hline Reziproke Translokationen & $0,5 \%(21)$ & $1,38 \%(323)$ & $1,51(86)$ \\
\hline Robertson-Translokationen & $0,36 \%(15)$ & $0,62 \%(145)$ & $0,66 \%(38)$ \\
\hline Inversionen & $0,14 \%(6)$ & $0,2 \%(47)$ & $0,21 \%(12)$ \\
\hline Geschlechtschromosomenfehlverteilung & $0,07 \%(3)$ & $0,06 \%(15)$ & $0,14 \%(8)$ \\
\hline Überzählige Chromosomen & $0,09 \%(4)$ & $0,03(6)$ & $0,02(1)$ \\
\hline $\begin{array}{l}\text { Gesamtzahl struktureller Chromosomenstö- } \\
\text { rungen (Translokationen und Inversionen) }\end{array}$ & $0,99 \%(42)$ & $2,2 \%(515)$ & $2,55 \%(136)$ \\
\hline
\end{tabular}

ändert, das genetische Material ist jedoch vollständig in normaler Kopienzahl vorhanden und funktionell intakt. So haben balancierte Translokationen zunächst einmal keinerlei klinisch relevante Auswirkungen für den Träger. Es können sich aber meiotische Fehlverteilungen ergeben, die in Abhängigkeit von den beteiligten Chromosomen das Risiko für Aborte oder für die Geburt eines Kindes mit einer Entwicklungsstörung aufgrund einer unbalancierten Chromosomenstörung erhöhen. Hieraus ergeben sich Konsequenzen für das Angebot einer pränatalen Diagnostik in weiteren Schwangerschaften.

Bei Paaren mit 2 oder mehr Aborten lässt sich in etwa $4-5 \%$ der Fälle bei einem Partner eine balancierte Chromosomenaberration nachweisen [7]. Die Inzidenz für eine strukturelle Chromosomenstörung pro Paar steigt von $0,7 \%$ in der Normalbevölkerung auf 2,0\% nach einem Abort, 4,4\% nach 2 Aborten und auf 5,1\% nach 3 Aborten (• Tab. 5). Das elterliche Geschlecht spielt bei den strukturellen Chromosomenstörungen keine entscheidende Rolle [25].
Chromosomenaberrationen bei Abortpaaren ausweisen, beruhen auf einer unzureichenden Berücksichtigung der Studienlage und zudem auf einer falschen Kalkulation der einzigen herangezogenen Studie [3]. In besagter Studie wurden 406 balancierte Chromosomenaberrationen bei 20.432 Personen nachgewiesen, dies entspricht einer Pro-Paar-Rate von etwa $4 \%$ und nicht - wie angegeben einer von $2 \%$.

\section{Mono- bzw. polygene Effekte}

Es liegen Ergebnisse zahlreicher genomweiter Assoziationsstudien vor, die mögliche maternale, paternale oder fetale genetische Effekte ausweisen, welche in der Summe aber bisher nur einen geringen Einfluss auf das Abortrisiko haben. Der Vergleich zwischen den Studien wird durch unterschiedliche Einschlusskriterien und unzureichende Kohortengrößen erschwert. Nach Einschätzung der DGGG/AIGM [20] ist nach derzeitigem Kenntnisstand eine molekulargenetische Analyse von Genvarianten, die bislang im Rahmen von Assoziationsstudien ermittelt wurden, bei Paaren mit wiederholten Aborten nicht sinnvoll.

Schwere monogene Entwicklungsstörungen können mit erhöhten Abortraten einhergehen und lassen sich nicht mit einer molekularzytogenetischen Diagnostik darstellen. Für die Diagnosestellung kommen molekulargenetische Techniken zum Einsatz (DNA-Sequenzierung, quantitative DNA-Analysen etc.), die das Vorhandensein einer adäquaten DNA-Probe und in der Regel eine bestimmte Verdachtsdiagnose voraussetzen. Insbesondere bei X-chromosomalen Krankheitsbildern mit Letalität im männlichen Geschlecht besteht ein erhöhtes Risiko für Fehlgeburten. Aber auch bei autosomal-dominanten und -rezessiven Krankheitsbildern, die schwere Fehlbildungen aufweisen, kann es zu einer erhöhten intrauterinen Mortalität kommen. Bei entsprechendem Verdacht ist eine klinisch-genetische und pathologische Untersuchung des Feten mit Asservierung von Blut oder Gewebe zur molekulargenetischen Diagnostik sinnvoll. Nur wenn die genaue genetische 
Ursache bekannt ist, kann im Rahmen einer humangenetischen Beratung eine Aussage über das Wiederholungsrisiko getroffen werden.

Eine generelle Untersuchung auf hereditäre Thrombophilien zum Zweck der Abortprophylaxe wird bei Frauen mit wiederholten Frühaborten nicht mehr empfohlen, weder in der deutschen Leitlinie [20], noch in anderen Leitlinien [1, 2, 31]. Die ESHRE-Leitlinie [11] sieht eine Diagnostik im Hinblick auf maternale hereditäre Thrombophilien nur unter wissenschaftlichen Aspekten als indiziert an. Die ASRM-Empfehlungen [2] schlagen eine Thrombophilieabklärung bei Frauen mit wiederholten Aborten ausschließlich bei positiver Eigen- oder Familienanamnese für thrombembolische Ereignisse vor. Die britische RCOGLeitlinie empfiehlt eine Analyse maternaler Thrombophiliefaktoren (FaktorV-Leiden-Variante, FVL, Prothombinvariante, Protein S-Mangel) nur bei Spätaborten (ab 2. Trimenon; [31]).

\section{》) Trotz zahlreicher Studien gibt es kein Indiz für eine Senkung der Frühabortrate durch Thromboseprophylaxe}

Nach zahlreichen ergebnislosen Interventionsstudien zur Reduktion der Abortraten bei Vorliegen einer maternalen Thrombophilie (Übersicht s. [20]) ist Ernüchterung eingetreten. Bis $\mathrm{zu}$ $15 \%$ der kaukasischen Bevölkerung weisen einen hereditären Thrombophiliefaktor auf: FVL (c.1601G >A in F5, rs6025), Prothrombin-G20210A-Variante (PT; c. ${ }^{*} 97 \mathrm{G}>\mathrm{A}$ in $\mathrm{F} 2, \mathrm{rs} 1799963$ ), Antithrombin-, Protein-C-, Protein-S-, Protein-Z- oder Faktor-XII-Mangel, Erhöhung von Faktor VIII oder Lipoprotein(a). Hinzu kommen Polymorphismen in den Genen der Methylentetrahydrofolatreduktase (MTHFR C677T bzw. c.665C>T), des Angiotensin-Converting-Enzyms (ACE) sowie des Plasminogen-Aktivator-Inhibitors (PAI), deren Prävalenz $>10 \%$ liegt. Solange es keine kontrollierten Studien gibt, die einen Nutzen einer Therapie eindeutig belegen können, ist eine antikoagulatorische
Behandlung von Frauen mit dem Ziel einer Abortreduktion als experimentell einzustufen.

\section{Strukturelle Chromosomen- aberration bei einem Elternteil und weitere Schwangerschaften}

Bei Vorliegen einer strukturellen Chromosomenaberration bei einem Elternteil ist die Wahrscheinlichkeit einer unbalancierten Chromosomenaberration im Abortmaterial oder beim Neugeborenen erhöht. In der ESHRE-Leitlinie [11] wird ein Risiko von 0,02\% für eine Lebendgeburt mit einer unbalancierten Chromosomenstörungen bei künftigen Schwangerschaften genannt. Dabei ist jedoch unklar, auf welcher Grundlage diese Zahl erhoben wurde. Größere Studien aus den 1980er-Jahren $[4,6]$ weisen eine Wahrscheinlichkeit von 2-5\% für die Geburt eines Kindes mit einer unbalancierten Chromosomenstörung aus. Durch eine verbesserte vorgeburtliche Diagnostik in den vergangenen Jahrzehnten hat sich diese Zahl vermutlich deutlich reduziert.

Paare mit nachgewiesenen strukturellen chromosomalen Auffälligkeiten, die durch wiederholte Fehlgeburten diagnostiziert wurden, weisen im Vergleich zu Paaren mit normalem Chromosomensatz eine signifikant höhere Anzahl von Fehlgeburten auf (ca. 50 vs. ca. 30\%) und verzichten häufiger auf weitere Schwangerschaften [13].

\section{Pränataldiagnostik}

Eine ursächliche Therapie von Chromosomenaberrationen ist nicht möglich. Im Laufe der Embryo- und Fetogenese wird ein Großteil der Fruchtanlagen mit Chromosomenstörungen durch ausbleibende Entwicklung oder Aborte „herausselektioniert“. Die meisten aneuploiden Embryonen kommen nicht zur Einnistung oder führen nur zu einer biochemischen Schwangerschaft bzw. zu einem Frühabort. Während 40-60\% der frühen Embryonen chromosomal auffällig sind, sinkt dieser Anteil auf 0,3\% bei Lebendgeburten [17].

Bei Nachweis einer balancierten elterlichen Chromosomenaberration steigt die Wahrscheinlichkeit einer meioti- schen Chromosomenfehlverteilung in der Keimbahn. Die Wahrscheinlichkeit einer lebensfähigen Chromosomenstörung bei künftigen Kindern ist im Falle einer reziproken Translokation invers mit der Größe der an der Translokation beteiligten Chromosomenabschnitte korreliert. Eine pränatale Chromosomenanalyse wird meist in Abhängigkeit vom Ausgangsrisiko nach individueller Abwägung mittels Chorionzottenbiopsie oder Amniozentese in weiteren (spontan eingetretenen) Schwangerschaften angeboten. Die Chorionzottenbiopsie hat den Vorteil, dass sie früher als die Amniozentese erfolgt (ab der 11.-12. Schwangerschaftswoche) und ein Ergebnis (Karyotyp) aus der Kurzzeitkultur bereits nach 1-2 Tagen vorliegt. Die Amniozentese wird meist zwischen der 16 . $(15+0)$ und 18. Schwangerschaftswoche durchgeführt. Eine Karyotypisierung ist erst nach 10-14 Tagen möglich, da die Amnionzellen erst kultiviert werden müssen. Der sog. FISH-Schnelltest wird direkt aus dem Fruchtwasser vorgenommen und erlaubt eine Aussage $\mathrm{zu}$ den wichtigsten Trisomien 13, 18, 21 bzw. Gonosomenaberrationen nach 1-2 Tagen. Mit beiden Methoden der invasiven Pränataldiagnostik ist ein Fehlgeburtenrisiko von $0,3-1 \%$ verbunden [21].

Je nach Größe und Lokalisation des chromosomalen Umbaus werden verschiedene Methoden verwendet. Während zur Erkennung submikroskopischer Umbauten molekularzytogenetische Untersuchungsmethoden(DNA-Arrayoder FISH-Analysen) zum Einsatz kommen, werden Robertson-Translokationen über konventionelle mikroskopische Chromosomenanalysen nachgewiesen. Unter Beteiligung eines Chromosoms 21 bei Robertson-Translokation kann mittels der Analyse zellfreier DNA aus dem mütterlichen Blut im Sinne eines nichtinvasiven Pränataltests (NIPT) bereits ab der 10.-11. Schwangerschaftswoche eine vergleichsweise sichere nichtinvasive Diagnostik angeboten werden. Das Fehlgeburtsrisiko einer invasiven Pränataldiagnostik kann durch einen Normalbefund bei einem NIPT vermieden werden. NIPT ist für die häufigsten Trisomien (Trisomie 13, 18, 21) welt- 
weit etabliert und zeigt einen hohen prädiktiven Wert.

Eine pränatale Diagnostik ist im deutschen Sprachraum bei familiären Chromosomenstörungen möglich, darf in allen 3 Ländern aber nur im Rahmen einer genetischen Beratung durch entsprechend qualifizierte Ärzte vorgenommen werden. Wird bei der pränatalen Diagnostik ein auffälliger Befund erhoben, kann eine medizinische Indikation für einen Schwangerschaftsabbruch bestehen, die sich in ihren "Maximalfristen“ deutlich von der „Fristenlösung“ unterscheidet. In diesen Fällen ist in allen 3 Ländern ein Schwangerschaftsabbruch im Prinzip bis zur Geburt des Kindes möglich.

Für den Abbruch von Schwangerschaften ohne medizinische Pathologien (oft als „Fristenlösung“ oder „Fristen- oder Beratungsregelung“ bezeichnet) gelten zeitliche Begrenzungen. In Deutschland ist ein Schwangerschaftsabbruch straffrei bis zum Abschluss der 14. Schwangerschaftswoche (post menstruationem, p. m.) und in der Schweiz bis zur vollendeten 12 . Schwangerschaftswoche (p.m.). In Österreich geht die Lehrmeinung von einer Fristenregelung bis zum Abschluss der 16. Schwangerschaftswoche (p.m.) aus.

\section{Präimplantations- und Präkonzeptionsdiagnostik}

Die Präimplantationsdiagnostik (PID) unterscheidet zwischen der gezielten Analyse vorbekannter genetischer Erkrankungen oder Risikokonstellationen in der Familie (monogene Erkrankungen oder balancierte Chromosomenveränderungen) und dem Screening auf embryonale Chromosomenfehlverteilungen zur Verbesserung der Schwangerschaftsraten im Rahmen der künstlichen Befruchtung. Im englischen Sprachraum werden für die gezielte PID mehrheitlich die Begriffe "preimplantation genetic testing“ (PGT) und „preimplantation genetic diagnosis“ (PGD) verwendet, für das chromosomale Screening die Bezeichnung „preimplantation genetic screening" (PGS).

Für die Darstellung des Chromosomensatzes wurde in der Vergangenheit meist mit der FISH gearbeitet, welche die gezielte Anfärbung bestimmter chromosomaler Bereiche mittels eines fluoreszierenden Farbstoffes erlaubt. In den letzten Jahren wurde die FISH-Analyse weitgehend durch die „comparative genomische Hybridisierung" (CGH) bzw. den DNAArray ersetzt. Diese Methoden erlauben den Nachweis von sehr kleinen Kopiezahlveränderungen im Genom. Mithilfe der neueren massiv-parallelen Sequenzierung („next generation sequencing“, NGS) können chromosomale Mosaike in einer höheren Sensitivität erkannt werden, wodurch sich im Vergleich zu anderen Methoden (z.B. DNA-Array) die Abortrate nach Transfer verringern soll [26].

\section{Gesetzliche Grundlagen}

Im Jahr 2014 ist die PID-Verordnung (PIDV) in Deutschland in Kraft getreten, welche die PID in engen Grenzen zulässt, wenn aufgrund der genetischen Disposition der Eltern oder eines Elternteils eine schwerwiegende Erbkrankheit beim Kind wahrscheinlich ist. Eine gezielte PID (PGD) bei bekanntem genetischen Risiko (Keimbahnmutation bei den Eltern) ist in Deutschland nur an hierfür zugelassenen PID-Zentren nach positivem Votum der zuständigen Ethikkommission erlaubt.

In Österreich wurde die rechtliche Voraussetzung für eine PID an embryonalen Zellen erst 2015 mit dem Fortpflanzungsmedizinrechts-Änderungsgesetz (FMedRÄG) geschaffen. Eine PID ist nur dann zulässig, wenn

1. keine Schwangerschaft nach 3 oder mehr Übertragungen entwicklungsfähiger Zellen eingetreten ist,

2. zumindest 3 ärztlich nachgewiesene Fehl- oder Totgeburten stattgefunden haben und

3. eine ernste Gefahr einer Fehl- oder Totgeburt oder einer Erbkrankheit des Kindes aufgrund der genetischen Disposition zumindest eines Elternteils besteht.

In der Schweiz trat erst am 01.09.2017 das revidierte Fortpflanzungsmedizingesetz zusammen mit der zugehörigen Verordnung in Kraft. Danach ist eine genetische Untersuchung von Embryonen
Hier steht eine Anzeige. Springer 
Tab. 6 Vergleich des Ausgangs von Schwangerschaften mit PGS und Spontanschwangerschaften bei Frauen mit mindestens 2 vorausgegangenen Aborten. Eingeschlossen wurden nur Paare mit balancierten Chromosomenveränderungen bei einem Partner

\begin{tabular}{|l|l|l|l|l|l|}
\hline Referenz & Kategorie & $\begin{array}{l}\text { Anzahl } \\
\text { Studien }\end{array}$ & $\begin{array}{l}\text { Anzahl } \\
\text { Patien- } \\
\text { ten }\end{array}$ & $\begin{array}{l}\text { Kumulative Le- } \\
\text { bendgeburten } \\
\text { (\% pro Paar) }\end{array}$ & $\begin{array}{l}\text { Aborte (\% } \\
\text { pro Paar) }\end{array}$ \\
\hline Musters et al. 2011 [27] & PGS & 5 & 181 & $64(35 \%)$ & $10(6 \%)$ \\
& Spontan-SS & 7 & 261 & $110(42 \%)$ & $72(28 \%)$ \\
\hline Franssen et al. 2011 [14] & PGS & 21 & 126 & $44(35 \%)$ & $6(5 \%)$ \\
& Spontan-SS & 2 & 299 & $238(80 \%)$ & $131(44 \%)$ \\
\hline Ikuma et al. 2015 [19] & PGS & 1 & 37 & $25(68 \%)$ & $9(24 \%)$ \\
& Spontan-SS & 1 & 52 & $34(65 \%)$ & $30(58 \%)$ \\
\hline
\end{tabular}

zulässig sowohl bei einer vererbbaren Veranlagung für eine schwere genetische Krankheit, die vor dem 50. Lebensjahr ausbricht, als auch zur Erkennung chromosomaler Eigenschaften, welche die Entwicklungsfähigkeit des Embryos beeinträchtigen können.

\section{Methoden der Zellgewinnung}

\section{Polkörperdiagnostik. Die Polkörper-} diagnostik (PKD) ist eine Methode zur genetischen Untersuchung von Eizellen noch vor Abschluss der Befruchtung (Präkonzeptionsdiagnostik). Die Entnahme und Untersuchung des ersten und zweiten Polkörpers ermöglicht eine indirekte Aussage über die genetische Konstitution der Eizelle. Ein grundsätzlicher Nachteil der Polkörperdiagnostik besteht darin, dass nur das mütterliche Erbgut untersucht wird und eine Aussage über mögliche paternale Faktoren nicht getroffen werden kann. Ferner ist zu bedenken, dass sich nur etwa $40-50 \%$ der befruchteten Eizellen überhaupt zu Blastozysten und nur $25 \%$ zu qualitativ mindestens guten Blastozysten entwickeln [34]. Damit lässt eine Chromosomenanalyse der Polkörper vielfach keine Aussage zur Prognose für die Weiterentwicklung des Embryos zu. Da die Polkörperbiopsie zeitlich vor der Verschmelzung der Vorkerne stattfindet, ist sie mit dem deutschen Embryonenschutzgesetz (ESchG) kompatibel und fällt nicht unter das österreichische FMedRÄG. Für die Durchführung der PKD steht jedoch nur ein enger zeitlicher Rahmen von maximal $20 \mathrm{~h}$ zwischen dem Eindringen des Spermiums und dem SichtbarWerden der Vorkerne zur Verfügung
[36]. Haupteinsatzgebiet der PKD ist die Aneuploidiediagnostik bei maternalen Risikofaktoren (Alter, Translokationen, X-chromosomale Erkrankungen). Paternale genetische Faktoren sind nicht, monogene Erkrankungen nur eingeschränkt diagnostizierbar. Aufgrund der höheren diagnostischen Aussagekraft hat sich international die PID gegenüber der PKD durchgesetzt. Interessant ist diesem Zusammenhang, dass sich der Gesetzgeber in Österreich ungeachtet der o. g. Einschränkungen erst kürzlich dafür ausgesprochen hat, die PID gegenüber der PKD grundsätzlich als nachrangig $\mathrm{zu}$ behandeln, d.h. eine PID hat $\mathrm{zu}$ unterbleiben, wenn mit hoher Wahrscheinlichkeit eine PKD zur Feststellung bzw. zum Ausschluss der genetischen Disposition ausreicht (Gentechnikbuch: 7. Kapitel, beschlossen am 20.01.2016).

Nach Auffassung der Autoren ist die PKD heutzutage nur noch dann sinnvoll, wenn das Paar dies ausdrücklich wünscht, z.B. um die Analyse an totiund pluripotenten Zellen zu vermeiden, bei monogenen Krankheiten und Vererbung über die Mutter und falls nur sehr wenige $(<6)$ Eizellen zur Befruchtung zur Verfügung stehen, sodass die zu erwartende Anzahl von Blastozysten mit ausreichend guter Qualität für eine geplante PID mittels Trophektodermbiopsie zu gering sein wird.

Blastomerbiopsie. Bei einer Blastomerbiopsie werden dem Embryo meist am 3. Tag im Achtzellstadium 1-2 totipotente Zellen entnommen. Durch die frühe Entnahme der Zellen können die Embryonen zeitnah im selben Zyklus transferiert werden. Allerdings führt die Biopsie zu einem relativen Rückgang der nachfolgenden Implantationsrate von $39 \%$ gegenüber nichtbiopsierten Embryonen [32]. Seit dieser Erkenntnis hat sich die Strategie der Zellentnahme sehr deutlich Richtung Blastozystenbiopsie entwickelt, aber die meisten Erfahrungen liegen für Blastomerbiopsien vor.

Eine entscheidende Einschränkung ist, dass der Chromosomenbefund einer untersuchten Zelle nicht für den Embryo repräsentativ ist, da in diesem Stadium über 50 \% der Embryonen chromosomale Mosaike, d.h. unterschiedliche Zelllinien mit normalem (euploiden) und nichtnormalem (aneuploiden) Chromosomensatz, aufweisen [10]. Rückschlüsse der entnommenen Zelle auf den sich in den ersten Zellteilungen befindlichen Embryo sind daher nur bedingt möglich. Im Falle eines falsch-positiven Befundes könnten Embryonen verworfen werden, obwohl die diagnostizierte Abweichung nur in einer Zelle vorhanden ist und sich im Laufe der embryonalen Entwicklung nicht mehr weiter entwickelt. Im Falle eines falsch-negativen Befundes könnten Embryonen eingesetzt werden, deren Chromosomenanomalie nicht erkannt wurde.

Blastozystenbiopsie. Bei einer Blastozystenbiopsie werden dem 5 Tage alten Embryo 5-7 pluripotente Trophoblastzellen entnommen. Diese Form der Biopsie scheint die Implantationsrate nicht negativ $\mathrm{zu}$ beeinflussen [32]. $\mathrm{Zu}$ diesem Zeitpunkt hat sich die innere Zellmasse, aus der sich der Embryo entwickelt, vom Trophektoderm, aus dem die spätere Plazenta und die Eihäute hervorgehen, differenziert. Die chromosomale Mosaikrate gegenüber den Blastomeren ist zwar geringer, liegt aber immer noch bei ca. $30 \%$ [26]. Auch hier gilt also, dass sich durch einen Mosaikbefund an Trophektodermzellen unter Umständen keine Aussage über den Chromosomenbefund des werdenden Kindes treffen lässt [37]. Berücksichtigt man die Tatsache, dass in Spontanaborten bis zur 12. Schwangerschaftswoche nur noch 5-6\% Mosaike nachgewiesen werden [23], ist anzunehmen, dass in den ersten Tagen der Embryonalent- 
Tab. 7 Empfehlungen verschiedener Fachgesellschaften für die Chromosomendiagnostik bei wiederholten Aborten (sortiert nach dem Jahr der Veröffentlichung)

\begin{tabular}{|c|c|c|c|c|c|}
\hline & ACOG 2002 [1] & RCOG 2011 [31] & ASRM 2012 [2] & ESHRE 2017 [11] & DGGG/AGIM [20] \\
\hline $\begin{array}{l}\text { Definition von wieder- } \\
\text { holten Aborten }\end{array}$ & $\begin{array}{l}\geq 2 \text { Aborte } \\
<15 \text { SSW }\end{array}$ & $\geq 3$ Aborte $\leq 24$ SSW & $\geq 2$ Aborte & $\geq 2$ Aborte $\leq 24$ SSW & $\geq 3$ Aborte $\leq 20$ SSW \\
\hline $\begin{array}{l}\text { Zytogenetische Unter- } \\
\text { suchung des Abortma- } \\
\text { terials }\end{array}$ & Nicht empfohlen & Empfohlen ab 3. Abort & Keine Angaben & Keine Angaben & Optional \\
\hline $\begin{array}{l}\text { Karyotypisierung der } \\
\text { Eltern }\end{array}$ & Empfohlen & $\begin{array}{l}\text { Nur bei Nachweis ei- } \\
\text { ner unbalancierten } \\
\text { strukturellen Chro- } \\
\text { mosomenstörung im } \\
\text { Abort }\end{array}$ & Empfohlen & $\begin{array}{l}\text { Nur bei erhöhtem ge- } \\
\text { netischen Risiko (nach } \\
\text { Geburt eines Kindes } \\
\text { mit Fehlbildungen oder } \\
\text { bekannter Chromo- } \\
\text { somenstörung in der } \\
\text { Familie) }\end{array}$ & Empfohlen \\
\hline $\begin{array}{l}\text { PGS bei weiteren } \\
\text { Schwangerschaften }\end{array}$ & Keine Angaben & Keine Angaben & Nicht empfohlen & Nicht empfohlen & Nicht empfohlen \\
\hline \multicolumn{6}{|c|}{$\begin{array}{l}\text { ACOG American College of Obstetricians and Gynecologists, RCOG Royal College of Obstetricians and Gynaecologists, ASRM American Society for } \\
\text { Reproductive Medicine, ESHRE European Society of Human Reproduction and Embryology, DGGG/AGIM Deutsche Gesellschaft für Gynäkologie und } \\
\text { Geburtshilfe/Arbeitsgemeinschaft für Immunologie in der Gynäkologie und Geburtshilfe, SSW Schwangerschaftswochen, PGS "preimplantation genetic } \\
\text { screening" }\end{array}$} \\
\hline
\end{tabular}

wicklung eine funktionelle Regulation von Aneuploidien stattfinden muss. Bis heute ist nicht geklärt, ob der Transfer von Embryonen mit nachgewiesenem Chromosomenmosaik tatsächlich zu einer schlechteren Lebendgeburtenrate führt im Vergleich zu einer Vorselektion von rein euploiden Embryonen [37]. Ein Nachteil der Blastozystenbiopsie ist, dass sich nur ca. $25 \%$ der befruchteten Eizellen zu morphologisch hochqualitativen Blastozysten am Tag 5 entwickeln. Bei dieser Methode liegen ferner nur wenige Stunden zwischen der Zellentnahme und dem Zeitpunkt, zu dem der Embryo wieder in den Uterus transferiert werden muss. Für die genetische Diagnostik im selben Zyklus besteht also nur ein sehr kleines Zeitfenster, da es sich die meisten Paare wünschen, noch im IVF(In-vitro-Fertilisation)-Behandlungszyklus einen Embryotransfer zu erhalten. In den meisten Fällen wird es aus logistischen Gründen nötig sein, die Embryonen nach der Biopsie am Tag 5 zu kryokonservieren, um sie dann für weitere Zyklen bereitzustellen. Aus technischer Sicht ist mit Kryoembryonen, die mit modernen und effizienten Methoden gefroren wurden (Vitrifikation), eine vergleichbare Schwangerschaftsrate $\mathrm{zu}$ erzielen wie mit Frischembryonen, sodass dieses Vorgehen technisch keinen Nachteil in sich birgt.
Bislang ist noch nicht geklärt, ob das Aneuploidiescreening im Rahmen der Sterilitätsbehandlung von Kinderwunschpaaren zu verbesserten Lebendgeburtenraten führt $[15,24]$. Hier sind weitere kontrollierte Studien unter Berücksichtigung von spezifischen Indikationen, eingesetzten Methoden und klar definierten Studienzielen erforderlich.

\section{PID/PGS bei Paaren mit wiederholten}

Aborten. Das Aneuploidiescreening (PGS) wird Sterilitätspaaren im Rahmen der Kinderwunschbehandlung durch IVF/ICSI (In-vitro-Fertilisation/ intrazytoplasmatische Spermieninjektion) mit dem Ziel angeboten, die Schwangerschaftsraten zu erhöhen. Im Unterschied zu Paaren mit Sterilität, die spontan nicht schwanger werden und deshalb eine künstliche Befruchtung (IVF/ICSI) in Anspruch nehmen, haben die meisten Paare mit einer Abortanamnese (Infertilität) eine hohe Wahrscheinlichkeit, erneut spontan schwanger zu werden. Da Frauen mit einer Abortanamnese meist eine normale Fruchtbarkeit aufweisen, ist die Wahrscheinlichkeit für einen raschen neuerlichen Schwangerschaftseintritt hoch. Die Chance auf ein lebend geborenes Kind ist bei einer spontan eingetretenen Schwangerschaft deutlich höher als nach künstlicher Befruchtung mit PID/PGS, wenngleich bei spontaner Konzeption eine erhöhte Abortrate in folgenden Schwangerschaften in Kauf genommen werden muss.

Durch das Verfahren der IVF/ICSI und den mit einer genetischen Analyse von embryonalen Zellen verbundenen Verlust von Embryonen liegt die Lebendgeburtenrate pro Zyklus nach künstlicher Befruchtung mit PGS bei etwa $35 \%$, während die Wahrscheinlichkeit einer Lebendgeburt im nächstfolgenden Zyklus einer spontan eingetretenen Schwangerschaft nach wiederholten Aborten etwa $60 \%$ beträgt [18].

Die bisherigen Studien geben keinen Hinweis darauf, dass durch PID/PGS nach IVF im Vergleich zu Spontanschwangerschaften bei Abortpaaren erhöhte Lebendgeburtenraten erzielt werden, auch nicht bei Paaren, die durch eine balancierte Chromosomenaberration bei einem Partner genetisch vorbelastet sind (•Tab. 6). Paare, die auf natürlichem Weg schwanger werden, nehmen allerdings eine deutlich höhere Abortrate in Kauf (28-58 \%) im Vergleich zu Paaren, die nach PID/PGS schwanger werden (5-24\%). Derzeit wird eine Präimplantationsdiagnostik bei Paaren mit wiederholten Aborten und balancierten Chromosomenstörungen von keiner Fachgesellschaft empfohlen (• Tab. 7). 


\section{Fazit für die Praxis}

- Bei Paaren mit mindestens 2 Aborten lässt sich in etwa 4-5\% der Fälle bei einem Partner eine balancierte strukturelle Chromosomenaberration nachweisen.

- Bei wiederholten Spontanaborten sollte eine Karyotypisierung bei beiden Partnern durchgeführt werden.

- Paaren mit einer balancierten Chromosomenaberration sollte in weiteren Schwangerschaften eine gezielte Pränataldiagnostik angeboten werden.

- Derzeit wird eine PID/PGS bei Paaren mit wiederholten Aborten von keiner Fachgesellschaft empfohlen.

- Die Chance auf ein lebend geborenes Kind ist in einer spontan eingetretenen Schwangerschaft deutlich höher als nach künstlicher Befruchtung mit PID/PGS, wenngleich bei spontaner Konzeption eine erhöhte Abortrate in folgenden Schwangerschaften in Kauf genommen werden muss.

- Bei Paaren, die aufgrund einer zusätzlichen Sterilität oder aus anderen Gründen eine künstliche Befruchtung in Anspruch nehmen, kann PID/PGS hilfreich sein, um die Chance auf eine Lebendgeburt pro Transfer zu erhöhen.

\section{Korrespondenzadresse}

\section{Prof. Dr. S. Rudnik-Schöneborn}

Sektion Humangenetik, Medizinische Universität Innsbruck

Peter-Mayr-Str. 1, 6020 Innsbruck, Österreich sabine.rudnik@i-med.ac.at

Funding. Open access funding provided by University of Innsbruck and Medical University of Innsbruck.

\section{Einhaltung ethischer Richtlinien}

Interessenkonflikt. S. Rudnik-Schöneborn, M. Swoboda und J. Zschocke geben an, dass kein Interessenkonflikt besteht.

Dieser Beitrag beinhaltet keine von den Autoren durchgeführten Studien an Menschen oder Tieren.

Open Access Dieser Artikel wird unter der Creative Commons Namensnennung 4.0 International Lizenz (http://creativecommons.org/licenses/by/4.0/deed. de) veröffentlicht, welche die Nutzung, Vervielfäl- tigung, Bearbeitung, Verbreitung und Wiedergabe in jeglichem Medium und Format erlaubt, sofern Sie den/die ursprünglichen Autor(en) und die Quelle ordnungsgemäßnennen, einen Link zur Creative Commons Lizenz beifügen und angeben, ob Änderungen vorgenommen wurden.

\section{Literatur}

1. American College of Obstetricians and Gynecologists (2002) ACOG practice bulletin. Management of recurrent pregnancy loss. Number 24, february 2001. (Replaces technical bulletin number 212, September 1995). American college of obstetricians and gynecologists. Int J Gynaecol Obstet 78:179-190

2. American Society of Reproductive Medicine (2012) ASRM Practice Committee. Evaluation and treatment of recurrent pregnancy loss: a committee opinion. Fertil Steril 98:1103-1111

3. Barber JC, Cockwell AE, Grant E et al (2010) Is karyotyping couples experiencing recurrent miscarriage worth the cost? BJOG 117:885-888

4. Boue A, Gallano P (1984) A collaborative study of the segregation of inherited chromosome structural rearrangements in 1356 prenatal diagnoses. Prenat Diagn 4:45-67

5. Branch DW, Gibson M, Silver RM (2010) Clinical practice. Recurrent miscarriage. N Engl J Med 363:1740-1747

6. Daniel A, Hook EB, Wulf G (1989) Risk of unbalanced progeny at amniocentesis to carriers of chromosome rearrangements: data from the United States and Canadian laboratories. Am J Med Genet 33:14-53

7. De Braekeleer M, Dao TN (1990) Cytogenetic studies in couples experiencing repeated pregnancy losses. Hum Reprod 5:519-528

8. Deutsche Gesellschaft für Humangenetik $(\mathrm{GfH})$, Berufsverband Deutscher Humangenetike (BVDH) (2011) S2-Leitlinie humangenetische Diagnostik und genetische Beratung. Medgen 23:281-323

9. Eiben B, Bartels I, Bähr-Porsch S et al (1990) Cytogeneticanalysis of 750 spontaneousabortions with the direct-preparation method of chorionic villi and its implications for studying genetic causes of pregnancy wastage. Am J Hum Genet 47:656-663

10. Esfandiari N, Bunnell ME, Casper RF (2016) Human embryo mosaicsim: did we drop the ball on chromosomal testing? J Assist Reprod Genet 33:1439-1444

11. ESHRE (2017) Recurrent pregnancy loss. Guideline of the European society of human reproduction and embryology

12. Franssen MT, Korevaar JC, Leschot NJ et al (2005) Selective chromosome analysis in couples with two or more miscarriages: case-control study. BM 331:137-141

13. Franssen MT, Korevaar JC, van der Veen F et al (2006) Reproductive outcome after chromosome analysis in couples with two or more miscarriages: index [corrected]-control study. BMJ 332:759-763

14. Franssen MT, Musters AM, van der Veen $F$ et al (2011) Reproductive outcome after PGD in couples with recurrent miscarriage carrying a structural chromosome abnormality: a systematic review. Hum Reprod Update 17:467-475

15. Gleicher N, Orvieto R (2017) Is the hypothesis of preimplantation genetic screening (PGS) still supportable? J Ovarian Res 10:21. https://doi.org/ 10.1186/s13048-017-0318-3
16. Goddijn M, Leschot NJ (2000) Genetic aspects of miscarriage. Baillieres Best Pract Res Clin Obstet Gynaecol 14:855-865

17. Hassold T, Hunt $P$ (2001) To err (meiotically) is human: the genesis of human aneuploidy. Nat Rev Genet 2:280-291

18. Hirshfeld-Cytron J, Sugiura-Ogasawara M, Stephenson MD (2011) Management of recurrent pregnancy loss associated with a parental carrier of a reciprocal translocation: a systematic review. Semin Reprod Med 29:470-481

19. Ikuma S, Sata T, Suguira-Ogasawara M et al (2015) Preimplantation genetic diagnosis and natural conception: a comparison of life birth rates in patients with recurrent pregnancy loss associated with translocation. PLoS ONE 10:e129958

20. Interdisziplinäre Leitlinie der Deutschen Gesellschaft für Gynäkologie und Geburtshilfe (DGGG) und der Arbeitsgemeinschaft Immunologie in der Gynäkologie und Geburtshilfe (AGIM) (2018) Diagnostik und Therapie von Frauen mit wiederholten Spontanaborten. S2k-Leitlinie

21. Kähler C, Gembruch U, Heling K-S et al (2013) Empfehlungen der DEGUM zur Durchführung von Amniozentese und Chorionzottenbiopsie. Ultraschall Med 34:435-440

22. Lund M, Kamper-Jørgensen M, Nielsen HS et al (2012) Prognosis for live birth in women with recurrent miscarriage: What is the best measure of success? Obstet Gynecol 119:37-43

23. Martinez MC, MéndezC, Ferro J et al (2010) Cytogenetic analysis of early nonviable pregnancies after assisted reproduction. Fertil Steril 93:289-292

24. MastenbroekS, Twisk M, van der Veen Fet al (2011) Preimplantation genetic screening: a systematic review and meta-analysis of RCTs. Hum Reprod Update 17:454-466

25. Meza-Espinoza JP, Anguiano LO, Rivera H (2008) Chromosomal abnormalities in couples with reproductive disorders. Gynecol Obstet Invest 66:237-240

26. Munne S, Wells D (2017) Detection of mosaicism at blastocyst stage with the use of highresolution next-generation sequencing. Fertil Steril 107:1085-1091

27. Musters AM, Repping S, Korevaar JC et al (2011) Pregnancy outcome after preimplantation genetic screening or natural conception in couples with unexplained recurrent miscarriage: a systematic review of the best available evidence. Fertil Steril 95:2153-2157

28. Nybo-Andersen AM, Wohlfahrt J, Christens $P$ et al (2000) Maternal age and fetal loss: population based register linkage study. BMJ 320:1708-1712

29. Ogasawara M, Aoki K, Okada S et al (2000) Embryonic karyotype of abortuses in relation to the number of previous miscarriages. Fertil Steril 73:300-304

30. Philipp T, Philipp K, Reiner A et al (2003) Embryoscopic and cytogenetic analysis of 233 missed abortions: factors involved in the pathogenesis of developmental defects of early failed pregnancies. Hum Reprod 18:1724-1732

31. RCOG (2011) The investigation and treatment of couples with recurrent first-trimester and secondtrimester miscarriage. In: College of Obstetricians Gynaecologists (Hrsg) RCOG green-top guideline no 17

32. Scott RT, Upham KM, Forman EJ et al (2013) Cleavage stage-stage biopsy significantly impairs human embryonic implantation potential while blastocyst biopsy does not: a randomized and paired clinical trial. Fertil Steril 100:624-630 
33. Soler A, Morales C, Mademont-Soler I et al (2017) Overview of chromosome abnormalities in first trimester miscarriages: a series of 1011 consecutive chorionic villi sample karyotypes. Cytogenet Genome Res 152:81-89

34. Thomas MR, Sparks AE, Ryan GL et al (2010) Clinical predictors of human blastocyste formation and pregnancy after extended embryo culture and transfer. Fertil Steril 94:543-548

35. Van den Berg MM, van Maarle MC, van Wely M et al (2012) Genetics of early miscarriage. Biochim Biophys Acta 1822:1951-1959

36. Van der Ven K, Montag M, van der Ven H (2008) Polkörperdiagnostik - ein Schritt in die richtige Richtung? Dtsch Arztebl 105:190-196

37. Vera-Rodriguez M, Rubio C (2017) Assessing the true incidence of mosaicism in preimplantation embryos. Fertil Steril 107:1107-1112

38. WHO (1977) Recommended definitions, terminology and format for statistical tables related to the perinatal period and use of a new certificate for cause of perinatal deaths. Modifications recommended by FIGO as amended October 14, 1976. Acta Obstet Gynecol Scand 56:247-253

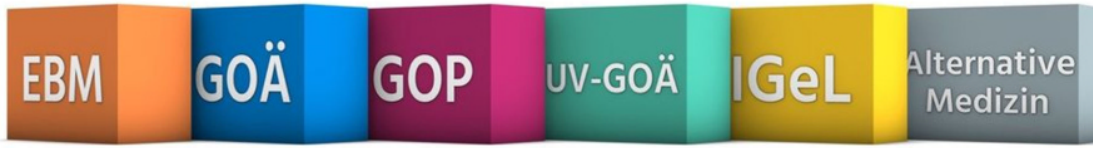

\section{Neue Abrechnungsdatenbank bei Springer Medizin: Alle relevanten Gebührenordnungen auf einen Klick}

Für Kommentare und Tipps zu Abrechnungsziffern dicke Bücher wälzen? Das muss nicht länger sein. Ärzte haben jetzt dank eines neuen Angebots von SpringerMedizin.de online Zugriff auf ihre wichtigsten Gebührenordnungen.

Mindestens drei Gebührenwerke sollte ein Facharzt präsent haben: den Einheitlichen Bewertungsmaßstab (EBM) für Kassenpatienten, die Gebührenordnung für Ärzte (GOÄ) für Privatversicherte und die UV-GOÄ für Patienten mit Berufs- oder Schulunfällen. Hinzu kommen Spezialfälle wie alternativmedizinisch behandelte Patienten oder Selbstzahler. Sicher, die Praxis-EDV hält das Regelwerk sämtlicher Gebührenordnungen vor, und auch die Kassenärztlichen Vereinigungen geben Hilfestellung bei der Quartalsabrechnung, doch im Zweifelsfall fehlt es oft an aktuellen Kommentierungen und an Tipps, wie die Abrechnung einer Leistung noch optimiert werden könnte oder vor welchen Fallen man sich hüten sollte.

\section{Kommentare, Urteile, Tipps}

Springer Medizin bietet jetzt eine Lösung für diese Problematik an: „Hermanns' kommentierte Datenbank zur Abrechnung". Sie ermöglicht die Recherche in den relevanten Gebührenordnungen EBM, GOÄ, UV-GOÄ und GOP. Das Besondere dabei: Den Ziffern sind über die reine Leistungsbeschreibung hinaus Kommentare, Urteile und Tipps beigefügt. Verlinkungen zwischen den einzelnen Positionen erlauben zudem einen schnellen Abgleich. Alle Angaben sind immer auf dem neuesten Stand, dafür sorgt Herausgeber Dr. Peter M. Hermanns mit einem Team von Abrechnungsexperten, und damit manchmal aktueller als die Praxis-EDV, bei der das letzte Update noch nicht gelaufen ist.

\section{Individuelle Pakete}

„Hermanns' kommentierte Datenbank zur Abrechnung" ist in die Plattform SpringerMedizin.de integriert und unter dem Menüpunkt
„GOÄ \& EBM“ zu finden. Der Zugriff ist für Ärzte, die bereits das Abonnement „e.Med Interdisziplinär" nutzen, frei, alle anderen können ein Paket ihrer Wahl buchen. Die Preise liegen zwischen $79 €$ pro Jahr für ein Gebührenwerk und $219 €$ pro Jahr für alle Teile: GOÄ, EBM, UV-GOÄ, GOP, IGeL und Alternative Medizin. Alle Änderungen in den gebuchten Gebührenordnungen werden ohne Aufpreis eingepflegt (ger).

Weitere Informationen sind abrufbar unter www. springermedizin.de/goae-ebm. Dort gibt es auch ein Video, das die neue Datenbank vorstellt.

Quelle: Springer Medizin

Mehr Informationen zu den e.MedAbonnements finden Sie unter: www.springermedizin.de/abo-shop 